\title{
带官能团的双有机磑化锌试剂与酰氯的反应研究
}

\author{
徐长明杨䂞黄丹凤* 牛腾胡雨来* 王克虎 \\ (西北师范大学化学化工学院 兰州 730070)
}

\begin{abstract}
摘要 探索了带醚键、酯基、羰基的双有机锌试剂的制备及其在 $\mathrm{CuCN} \cdot 2 \mathrm{LiCl}$ 存在下和酰氯的反应，得到了带官能团 的二酮类化合物. 该方法条件温和，操作简便，产率中等，提供了合成带官能团的长链二酮类化合物的一种简便方法. 关键词 双有机碘化锌试剂; 酰氯; 二酮类化合物
\end{abstract}

\section{Study on the Coupling Reaction of Fuctionalized Bisorganozinc lodides and Acyl Chlorides}

\author{
Xu, Changming Yang, Lei Huang, Danfeng* Niu, Teng Hu, Yulai* Wang, Kehu \\ (College of Chemistry and Chemical Engineering, Northwest Normal University, Lanzhou 730070)
}

\begin{abstract}
This paper investigated the preparation of fuctionalized bisorganozinc iodides with ether, ester, carbonyl groups and the reactions with acyl chlorides in the presence of $\mathrm{CuCN} \cdot 2 \mathrm{LiCl}$ generating fuctionalized diketones. The method has the advantages of mild and simple reaction conditions with medium yields, and provides a simple synthesis procedure of fuctionalized long-chain diketone compounds.
\end{abstract}

Keywords bisorganozinc iodide; acyl chloride; diketone compound

有机金属试剂是有机合成中构建碳碳键最有效和 应用最广泛的试剂之一. 1849 年, Frankland ${ }^{[1]}$ 首先制备 得到了 $\mathrm{Et}_{2} \mathrm{Zn}$, 但由于其化学反应活性不高, 故在当时 没有引起足够的重视. 截至 1880 年, 人们只研究了此类 有机锌试剂与典型的亲电试剂如酰氯、醛、酮、酯反应 形成碳碳键的反应 ${ }^{[2]}$. 然而由于其活性低, 在 20 世纪初 被反应活性更高的有机镁化合物取代了，只有 Reformasky 试剂在有机合成中继续应用 ${ }^{[3]}$. 但是高活性 有机金属试剂(如有机锂试剂和有机镁试剂)的化学选择 性相应地就比较差, 同时在其分子中不允许有活性官能 团(如羰基、酯基等)存在. 因此在使用有机金属试剂前 必须要先将活性官能团保护起来, 反应完成后再将保护 基去掉, 这样延长了合成路线, 降低了合成效率. 随着 有机合成化学的发展, 人们的兴趣又逐渐转向低活性的 有机金属试剂, 因为它们具有较好的官能团相容性和化 学反应选择性, 并且通过金属转移、催化剂催化等手段 可提高此类试剂的活性 ${ }^{[4]}$, 这就很好地解决了试剂的活
性与试剂的官能团相容性这一对矛盾. 有机锌试剂就是 这类试剂中应用比较广泛的有机金属试剂之一.

1936年 Hunsdicker 等 ${ }^{[5}$ 制得了第一个带有官能团的 有机锌试剂 $\mathrm{RO}_{2} \mathrm{C}\left(\mathrm{CH}_{2}\right)_{n} \mathrm{ZnI}$. 三十多年以后, Wittig 和 Jautelat ${ }^{[6]}$ 又报道了 $\mathrm{PhCO}_{2} \mathrm{CH}_{2} \mathrm{ZnI}$ 及 $\mathrm{PhCO}_{2}\left(\mathrm{CH}_{2}\right)_{3} \mathrm{ZnI}$ 的 制备. 1978 年 Mukaiyama 等 ${ }^{[7]}$ 首次报道了在 $\beta$-氨基醇的 存在下 $\mathrm{Et}_{2} \mathrm{Zn}$ 可与苯甲醛顺利地发生加成反应生成产率 为 $76 \%$ 的 1,2-加成产物. 从此以后，人们对有机锌在有 机合成中的应用作了大量的研究，但大部分的工作都集 中在二烷基锌与醛、酮的加成反应上 ${ }^{[8]} .1980$ 年 Negishi 等 ${ }^{[9]}$ 实现了 $\mathrm{Pd}$ 络合物催化 $\mathrm{RZnX}$ 试剂与卤化物或酰氯 的偶联反应. 1988 年, Knochel 等 ${ }^{[4]}$ 报道了 $\mathrm{CuCN} \cdot 2 \mathrm{LiCl}$ 存在下 $\mathrm{RZnX}$ 与酰氯的偶联反应和与 $\alpha, \beta$-不饱和醛、酮 的 1,4-加成反应，反应中有机锌试剂所带的官能团(羰 基、卤素、酯基)等能够被保留. 在 1991 年 Knochel 等 ${ }^{[10]}$ 又首次报道了不带官能团的双有机卤化锌试剂的制备 及应用. 最近几年我们实验室报道了 $\mathrm{RZnX}$ 与醛酮的加

*E-mail: huangdf@nwnu.edu.cn; huyl@nwnu.edu.cn

Received September 6, 2011; revised October 27, 2011; published online November 16, 2011.

Project supported by the National Natural Science Foundation of China (No. 2072068), the Natural Science Foundation of Gansu Province (No. 3ZS041-A25-003), and the Specialized Research Fund of the Doctoral Propram of Higher Education (No. 200807360001).

国家自然科学基金(No. 20472068)、甘肃省自然科学基金(No. 3ZS041-A25-003)、教育部高等学校博士学科点专项科研基金(No. 200807360001)资助项 目. 
成-取代、加成-消去反应，以及与硝基烯烃的 1,4-加成 反应 ${ }^{[11]}$. 另外, 我们实验室也报道了不带官能团的双烷 基锌试剂与酰氯的偶联反应 ${ }^{[12]}$. 为了扩展有机锌试剂 在有机合成中的应用, 我们又首次制备了含有醚键 (Scheme 1)、酯基 (Scheme 2) 和羰基 (Scheme 3)的双有 机锌试剂, 并且成功实现了其在 $\mathrm{CuCN} \cdot 2 \mathrm{LiCl}$ 存在下和 酰氯的反应, 得到带官能团的二酮类化合物, 提供了一 种合成带官能团的二酮类化合物的简便方法.

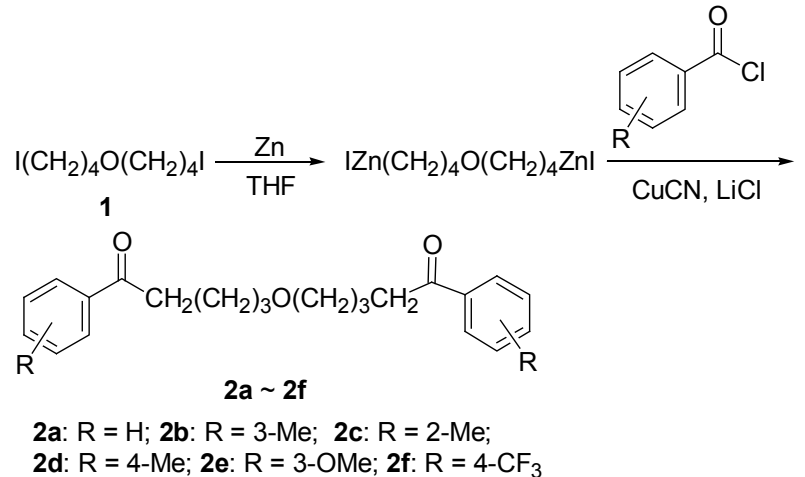

Scheme 1
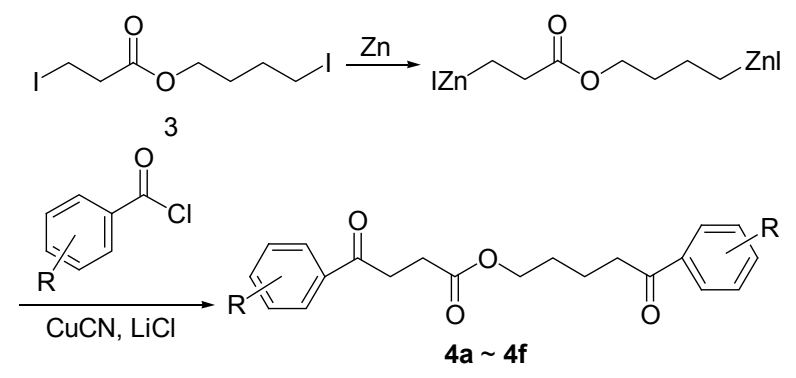

4a: $\mathrm{R}=\mathrm{H} ;$ 4b: $\mathrm{R}=3-\mathrm{Me} ; 4 \mathrm{c}: \mathrm{R}=4-\mathrm{Me}$

4d: $R=3-O M e ; 4 e: ~ R=4-C_{3} ; 4 f: R=4-C l$

Scheme 2

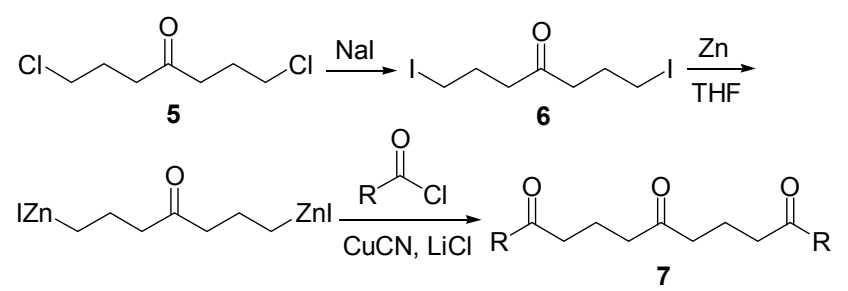

7a: $\mathrm{R}=\mathrm{Ph} ; \mathbf{7 b}: \mathrm{R}=4-\mathrm{MeC}_{6} \mathrm{H}_{4} ; \mathbf{7 c}: \mathrm{R}=3-\mathrm{MeC}_{6} \mathrm{H}_{4} ; \mathbf{7 d}: \mathrm{R}=2-\mathrm{MeC}_{6} \mathrm{H}_{4}$ 7e: $\mathrm{R}=4-\mathrm{ClC}_{6} \mathrm{H}_{4} ; 7 \mathrm{f}: \mathrm{R}=4-\mathrm{CF}_{3} \mathrm{C}_{6} \mathrm{H}_{4} ; 7 \mathrm{~g}: \mathrm{R}=2-$ Furyl; $7 \mathrm{~h}: \mathrm{R}=\mathrm{Et}$

Scheme 3

\section{1 实验部分}

\section{1 仪器与试剂}

红外光谱仪为 Alpha Centauri FI-IR 型仪器, 固体采 用 $\mathrm{KBr}$ 压片法, 液体采用液膜法. 核磁共振谱用 BRUKER PT jxf790425AM $400 \mathrm{MHz}$ 型核磁共振仪测定, 以気代氯仿作为溶剂, TMS 为内标. 质谱用 $\mathrm{QP}-1000 \mathrm{~A}$
GC-MS 测定, EI源. 元素分析用 Carlo Erba-1106 测定. 熔 点测定用显微熔点测定仪测定, 温度计未校正. 化合物 1, 3, 5, 6 按照文献 $[12 ， 13]$ 方法自制, 其它试剂均为国产 分析纯级.

\section{2 二酮类化合物的合成}

在干燥的 $25 \mathrm{~mL}$ 两颈瓶中加入活化的锌粉 (12 $\mathrm{mmol})^{[14]}$, 用氮气置换瓶中的空气后, 加入二碘代烃 $(6$ $\mathrm{mmol})$ 及 $\mathrm{THF}(10 \mathrm{~mL})$, 在 $40 \sim 45{ }^{\circ} \mathrm{C}$ 下搅拌反应 $10 \sim 12$ $\mathrm{h}$ ，得双有机硒化锌的 THF 溶液.

在另一只 $100 \mathrm{~mL}$ 干燥的三颈瓶中加入 $\mathrm{CuCN}(12$ $\mathrm{mmol})$ 和 $\mathrm{LiCl}(24 \mathrm{mmol})$, 在 $100 \sim 110{ }^{\circ} \mathrm{C}$ 下真空 $(5$ $\mathrm{mmHg})$ 干燥 $1 \mathrm{~h}$, 用氮气置换瓶中的空气，加入 THF (10 $\mathrm{mL}$ ), 室温摚拌 $30 \mathrm{~min}$, 反应混合物变成灰绿色, 将反 应混合物冷到 $-25{ }^{\circ} \mathrm{C}$, 加入上面制得的双有机碘化锌 的 THF 溶液, 在 $0{ }^{\circ} \mathrm{C}$ 下搅拌 $15 \mathrm{~min}$, 再将反应混合物冷 到 $-25{ }^{\circ} \mathrm{C}$, 滴加酰氯 $(9.6 \mathrm{mmol})$, 加完后搅拌反应混合 物，自然升温. TLC 检测酰氯反应完全后，向反应混合 物中加入 $30 \mathrm{~mL}$ 饱和氯化铵水溶液, 搅拌 $15 \mathrm{~min}$, 再加 入 $15 \mathrm{~mL}$ 乙醚, 萃取, 分出有机相, 水相再用乙醚萃取, 合并有机相，用无水硫酸镁干燥，蒸去溶剂后，进行柱 层析分离得到纯品.

二(4-苯甲酰基)正丁醚(2a)：白色晶体，产率 54\%. m.p. $42 \sim 43{ }^{\circ} \mathrm{C} ;{ }^{1} \mathrm{H}$ NMR $\left(400 \mathrm{MHz}, \mathrm{CDCl}_{3}\right) \delta$ : 7.95 (t, $J=7.6 \mathrm{~Hz}, 4 \mathrm{H}), 7.54(\mathrm{~d}, J=6.8 \mathrm{~Hz}, 2 \mathrm{H}), 7.45$ (t, $J=7.6$ $\mathrm{Hz}, 4 \mathrm{H}), 3.46$ (t, $J=6.4 \mathrm{~Hz}, 4 \mathrm{H}), 3.00$ (t, $J=7.2 \mathrm{~Hz}, 4 \mathrm{H}$ ), $1.82(\mathrm{t}, J=6.8 \mathrm{~Hz}, 4 \mathrm{H}), 1.68 \sim 1.63(\mathrm{~m}, 4 \mathrm{H}) ;{ }^{13} \mathrm{C}$ NMR $\left(100 \mathrm{MHz}, \mathrm{CDCl}_{3}\right) \delta: 200.2,136.9,132.9,128.5,128.0$, 70.6, 38.2, 29.3, 21.0; IR (KBr) v: 3058, 2953, 2920, 2854, 1682, 1355, 1116, 758, $689 \mathrm{~cm}^{-1}$; MS m/z: 77 (44), 105 (100), 160 (43), 161 (16), 261 (0.11). Anal. calcd for $\mathrm{C}_{22} \mathrm{H}_{26} \mathrm{O}_{3}$ : C 78.07, H 7.74; found C 78.13, H 7.70.

二[4-(3'-甲基)苯甲酰基]正丁醚(2b): 白色晶体，产 率 55\%. m.p. $38 \sim 39{ }^{\circ} \mathrm{C} ;{ }^{1} \mathrm{H}$ NMR $\left(400 \mathrm{MHz}, \mathrm{CDCl}_{3}\right) \delta$ : 7.75 (t, $J=7.6 \mathrm{~Hz}, 4 \mathrm{H}), 7.35$ (t, $J=1.2 \mathrm{~Hz}, 4 \mathrm{H}), 3.45$ (t, $J=6.0 \mathrm{~Hz}, 4 \mathrm{H}), 2.98(\mathrm{t}, J=7.6 \mathrm{~Hz}, 4 \mathrm{H}), 2.41(\mathrm{~s}, 6 \mathrm{H})$, $1.82 \sim 1.77(\mathrm{~m}, 4 \mathrm{H}), 1.69 \sim 1.63(\mathrm{~m}, 4 \mathrm{H}) ;{ }^{13} \mathrm{C}$ NMR $(100$ $\left.\mathrm{MHz}, \mathrm{CDCl}_{3}\right) \delta: 200.4,138.3,133.6,128.5,128.4,125.2$, 70.6, 38.3, 29.3, 21.3, 21.1; IR (KBr) v: 3053, 2945, 2920, 2864, 1685, 1156, 1107, 779, 740, $687 \mathrm{~cm}^{-1}$; MS m/z: 366 $\left(\mathrm{M}^{+}, 0.4\right), 65$ (15), 91 (55), 119 (100), 174 (45). Anal. calcd for $\mathrm{C}_{24} \mathrm{H}_{30} \mathrm{O}_{3}$ : C 78.65, H 8.25; found C 78.60, H 8.28 .

二[4-(2'-甲基)苯甲酰基]正丁醚(2c): 无色油状液 体, 产率 52\%. ${ }^{1} \mathrm{H}$ NMR $\left(400 \mathrm{MHz}, \mathrm{CDCl}_{3}\right) \delta: 7.61(\mathrm{~d}, J=$ $7.6 \mathrm{~Hz}, 2 \mathrm{H}), 7.35$ (t, $J=7.6 \mathrm{~Hz}, 2 \mathrm{H}), 7.24$ (t, $J=7.6 \mathrm{~Hz}$, 
4H), 3.44 (t, $J=6.4 \mathrm{~Hz}, 4 \mathrm{H}), 2.91(\mathrm{t}, J=6.8 \mathrm{~Hz}, 4 \mathrm{H}), 2.48$ $(\mathrm{s}, 6 \mathrm{H}), 1.79 \sim 173(\mathrm{~m}, 4 \mathrm{H}), 1.67 \sim 1.60(\mathrm{~m}, 4 \mathrm{H}) ;{ }^{13} \mathrm{C} \mathrm{NMR}$ $\left(100 \mathrm{MHz}, \mathrm{CDCl}_{3}\right) \delta: 204.5,138.1,137.8,131.8,131.0$, 128.3, 125.6, 70.6, 41.3, 29.3, 21.2, 21.1; IR v: 3063, 3021, 2933, 2862, 1686, 1452, 1115, 967, 754, $652 \mathrm{~cm}^{-1}$; MS m/z: $366\left(\mathrm{M}^{+}, 0.33\right), 65$ (15), 91 (57), 119 (100), 174 (21). Anal. calcd for $\mathrm{C}_{24} \mathrm{H}_{30} \mathrm{O}_{3}$ : C 78.65, $\mathrm{H}$ 8.25; found $\mathrm{C} 78.70$, H 8.21.

二[4-(4'-甲基)苯甲酰基]正丁醚(2d): 白色晶体，产 率 53\%. m.p. 56 57 ${ }^{\circ} \mathrm{C} ;{ }^{1} \mathrm{H}$ NMR $\left(400 \mathrm{MHz}, \mathrm{CDCl}_{3}\right) \delta$ : $7.85(\mathrm{~d}, J=2.0 \mathrm{~Hz}, 4 \mathrm{H}), 7.26 \sim 7.24(\mathrm{~m}, 4 \mathrm{H}), 3.45(\mathrm{t}, J=$ $6.4 \mathrm{~Hz}, 4 \mathrm{H}), 2.97$ (t, $J=7.6 \mathrm{~Hz}, 4 \mathrm{H}), 2.40(\mathrm{~s}, 6 \mathrm{H}), 1.80(\mathrm{t}$, $J=7.2 \mathrm{~Hz}, 4 \mathrm{H}), 1.67 \sim 1.63(\mathrm{~m}, 4 \mathrm{H}) ;{ }^{13} \mathrm{C} \mathrm{NMR}(100 \mathrm{MHz}$, $\left.\mathrm{CDCl}_{3}\right) \delta: 199.9,143.6,134.5,129.2,128.1,70.6,38.1$, 29.3, 21.6, 21.2; IR (KBr) v: 3034, 2937, 2867, 2801, 1676, 1604, 1115, 799, $763 \mathrm{~cm}^{-1}$; MS m/z: $366\left(\mathrm{M}^{+}, 0.32\right)$, 65 (14), 91 (45), 119 (100), 174 (38). Anal. calcd for $\mathrm{C}_{24} \mathrm{H}_{30} \mathrm{O}_{3}$ : C 78.65, $\mathrm{H}$ 8.25; found C 78.61, H 8.30.

二[4-(3'-甲氧基)苯甲酰基]正丁醚(2e): 白色晶体, 产率 52\%. m.p. 56 57 ${ }^{\circ} \mathrm{C} ;{ }^{1} \mathrm{H}$ NMR $\left(400 \mathrm{MHz}, \mathrm{CDCl}_{3}\right) \delta$ : 7.53 (d, $J=8.0 \mathrm{~Hz}, 2 \mathrm{H}), 7.48$ (d, $J=2.0 \mathrm{~Hz}, 2 \mathrm{H}), 7.36$ (t, $J=8.0 \mathrm{~Hz}, 2 \mathrm{H}), 7.09(\mathrm{dd}, J=2.4,8.4 \mathrm{~Hz}, 2 \mathrm{H}), 3.85$ (s, $6 \mathrm{H}), 3.45$ (t, $J=6.0 \mathrm{~Hz}, 4 \mathrm{H}), 2.98$ (t, $J=7.2 \mathrm{~Hz}, 4 \mathrm{H}), 1.81$ $(\mathrm{t}, J=7.2 \mathrm{~Hz}, 4 \mathrm{H}), 1.67 \sim 1.63(\mathrm{~m}, 4 \mathrm{H}) ;{ }^{13} \mathrm{C}$ NMR $(100$ $\left.\mathrm{MHz} \mathrm{CDCl}_{3}\right) \delta: 200.0,159.8,138.3,129.5,120.6,119.3$, 112.2, 70.5, 55.4, 38.3, 29.3, 21.1; IR (KBr) v: 3075, 3007, 2945, 2865, 1682, 1594, 1265, 1163, 1107, 783, 747, 680 $\mathrm{cm}^{-1}$; MS m/z: 77 (25), 92 (20), 107 (28), 135 (100), 190 (66), 191 (26), 207 (0.31). Anal. calcd for $\mathrm{C}_{24} \mathrm{H}_{30} \mathrm{O}_{5}$ : C 72.34, H 7.59; found C 72.28, H 7.55.

二[4-(4'-三氟甲基)苯甲酰基]正丁醚(2f)：白色晶体， 产率 66\%. m.p. 78 $79{ }^{\circ} \mathrm{C} ;{ }^{1} \mathrm{H}$ NMR $\left(400 \mathrm{MHz}, \mathrm{CDCl}_{3}\right) \delta$ : 7.06 (d, $J=8.4 \mathrm{~Hz}, 4 \mathrm{H}), 7.73$ (d, $J=8.0 \mathrm{~Hz}, 4 \mathrm{H}), 3.46$ (d, $J=5.6 \mathrm{~Hz}, 4 \mathrm{H}), 3.03(\mathrm{t}, J=7.6 \mathrm{~Hz}, 4 \mathrm{H}), 1.87 \sim 1.80(\mathrm{~m}$, $4 \mathrm{H}), 1.69 \sim 1.62(\mathrm{~m}, 4 \mathrm{H}) ;{ }^{13} \mathrm{C}$ NMR $\left(100 \mathrm{MHz}, \mathrm{CDCl}_{3}\right) \delta$ : $199.0,139.5,134.4,134.0,128.3,125.6,125.5,124.9$, 70.5, 38.5, 29.1, 20.9; IR (KBr) v: 2940, 2866, 1684, 1329, 1130, 1012, 976, 753, 729, $602 \mathrm{~cm}^{-1}$; MS m/z: $474\left(\mathrm{M}^{+}\right.$, 0.02), 41 (9.5), 55 (7.1), 95 (5.0), 145 (46), 173 (100), 228 (20). Anal. calcd for $\mathrm{C}_{24} \mathrm{H}_{24} \mathrm{~F}_{6} \mathrm{O}_{3}: \mathrm{C} 60.76, \mathrm{H}$ 5.10; found $\mathrm{C}$ 60.73, H 5.06 .

4-苯基-4-丁酮酸(4-苯甲酰基)丁酯(4a)：白色晶体， 产率 52\%. m.p. $79 \sim 80{ }^{\circ} \mathrm{C} ;{ }^{1} \mathrm{H}$ NMR $\left(400 \mathrm{MHz}, \mathrm{CDCl}_{3}\right) \delta$ : $7.98 \sim 7.91(\mathrm{~m}, 4 \mathrm{H}), 7.57 \sim 7.53(\mathrm{~m}, 2 \mathrm{H}), 7.50 \sim 7.27(\mathrm{~m}$, $4 \mathrm{H}), 4.17 \sim 4.12(\mathrm{~m}, 2 \mathrm{H}), 3.32 \sim 3.27(\mathrm{~m}, 2 \mathrm{H}), 3.00 \sim 2.97$ $(\mathrm{m}, 2 \mathrm{H}), 2.77 \sim 2.72(\mathrm{~m}, 2 \mathrm{H}), 1.85 \sim 1.78(\mathrm{~m}, 2 \mathrm{H}), 1.77 \sim$ $1.70(\mathrm{~m}, 2 \mathrm{H}) ;{ }^{13} \mathrm{C}$ NMR $\left(100 \mathrm{MHz}, \mathrm{CDCl}_{3}\right) \delta$ : 199.6, $197.9,172.8,136.7,136.4,133.1,132.9,128.4,127.9$, $127.8,64.2,37.7,33.2,28.1,28.0,20.4$; IR (KBr) v: 3063, 2957, 2908, 1726, 1684, 1215, 1166, 964, 756, $691 \mathrm{~cm}^{-1}$; MS m/z: 51 (10), 77 (40), 105 (100), 160 (9), 161 (0.95), 177 (0.93). Anal. calcd for $\mathrm{C}_{21} \mathrm{H}_{22} \mathrm{O}_{4}$ : C 74.54, $\mathrm{H}$ 6.55; found C $74.58, \mathrm{H} 6.49$.

4-(3'-甲基)苯基-4-丁酮酸[4-(3'-甲基)苯甲酰基]丁 酯(4b): 白色晶体，产率 58\%. m.p. 57 58 ${ }^{\circ} \mathrm{C} ;{ }^{1} \mathrm{H}$ NMR $\left(400 \mathrm{MHz}, \mathrm{CDCl}_{3}\right) \delta: 7.76(\mathrm{t}, J=8.8 \mathrm{~Hz}, 4 \mathrm{H}), 7.33(\mathrm{t}, J=$ $7.2 \mathrm{~Hz}, 4 \mathrm{H}), 4.16 \sim 4.10(\mathrm{~m}, 2 \mathrm{H}), 3.31 \sim 3.25(\mathrm{~m}, 2 \mathrm{H})$, $3.00 \sim 2.94(\mathrm{~m}, 2 \mathrm{H}), 2.76 \sim 2.70(\mathrm{~m}, 2 \mathrm{H}), 2.40(\mathrm{~s}, 6 \mathrm{H})$, $1.83 \sim 1.72(\mathrm{~m}, 4 \mathrm{H}) ;{ }^{13} \mathrm{C}$ NMR $\left(100 \mathrm{MHz}, \mathrm{CDCl}_{3}\right) \delta$ : $199.8,198.1,172.8,138.2,136.8,136.4,133.7,128.4$, $125.1,64.2,37.7,33.3,28.1,28.0,21.2,20$; IR (KBr) $v$ : 3026, 2949, 1726, 1678, 1224, 1155, 780, 685, $538 \mathrm{~cm}^{-1}$; MS m/z: $366\left(\mathrm{M}^{+}, 0.02\right), 65$ (13), 91 (44), 119 (100), 175 (25), 191 (0.3). Anal. calcd for $\mathrm{C}_{23} \mathrm{H}_{26} \mathrm{O}_{4}$ : C 75.38, H 7.15; found C 75.41, H 7.12.

4-(4'-甲基)苯基-4-丁酮酸[4-(4'-甲基)苯甲酰基]丁 酯(4c): 白色晶体，产率 56\%. m.p. 97 98 ${ }^{\circ} \mathrm{C} ;{ }^{1} \mathrm{H}$ NMR $\left(400 \mathrm{MHz}, \mathrm{CDCl}_{3}\right) \delta: 7.87(\mathrm{t}, J=8.0 \mathrm{~Hz}, 4 \mathrm{H}), 7.25$ (d, $J=$ $7.6 \mathrm{~Hz}, 4 \mathrm{H}), 4.15(\mathrm{t}, J=6.0 \mathrm{~Hz}, 2 \mathrm{H}), 3.29$ (t, $J=6.8 \mathrm{~Hz}$, 2H), 2.98 (t, $J=6.8 \mathrm{~Hz}, 2 \mathrm{H}), 2.74(\mathrm{t}, J=6.8 \mathrm{~Hz}, 2 \mathrm{H}), 2.40$ $(\mathrm{s}, 6 \mathrm{H}), 1.83 \sim 1.71(\mathrm{~m}, 4 \mathrm{H}) ;{ }^{13} \mathrm{C}$ NMR $\left(100 \mathrm{MHz}, \mathrm{CDCl}_{3}\right)$ $\delta: 199.4,197.7,173.0,143.9,143.7,134.4,134.0,129.2$, 128.1, 64.3, 37.7, 33.2, 28.3, 28.1, 21.6, 20.6; IR (KBr) v: 3038, 2933, 1733, 1674, 1606, 1316, 1168, 980, 791, 567 $\mathrm{cm}^{-1}$; MS m/z: 65 (10), 91 (34), 119 (100), 174 (17), 175 (16), 191 (0.91). Anal. calcd for $\mathrm{C}_{23} \mathrm{H}_{26} \mathrm{O}_{4}: \mathrm{C} 75.38, \mathrm{H}$ 7.15; found C 75.35, H 7.20.

4-(3'-甲氧基)苯基-4-丁酮酸[4-(3'-甲氧基)苯甲酰 基]丁酯(4d)：白色晶体，产率 52\%. m.p. 48 49 ${ }^{\circ} \mathrm{C} ;{ }^{1} \mathrm{H}$ NMR (400 MHz, $\left.\mathrm{CDCl}_{3}\right) \delta: 7.57 \sim 7.48(\mathrm{~m}, 4 \mathrm{H}), 7.39 \sim$ $7.34(\mathrm{~m}, 2 \mathrm{H}), 7.12 \sim 7.09(\mathrm{~m}, 2 \mathrm{H}), 4.15(\mathrm{t}, J=6.4 \mathrm{~Hz}, 2 \mathrm{H})$, $3.86(\mathrm{~s}, 6 \mathrm{H}), 3.30(\mathrm{t}, J=6.4 \mathrm{~Hz}, 2 \mathrm{H}), 3.00(\mathrm{t}, J=6.8 \mathrm{~Hz}$, $2 \mathrm{H}), 2.75(\mathrm{t}, J=6.8 \mathrm{~Hz}, 2 \mathrm{H}), 1.85 \sim 1.72(\mathrm{~m}, 4 \mathrm{H}) ;{ }^{13} \mathrm{C}$ NMR $\left(100 \mathrm{MHz}, \mathrm{CDCl}_{3}\right) \delta: 199.5,197.9,172.9,159.7$, $138.2,137.8,129.5,120.6,119.7,119.4,112.2,112.1$, 64.3, 55.4, 37.9, 33.4, 28.2, 28.1, 20.6; IR (KBr) v: 3070, 2947, 1732, 1681, 1593, 1429, 1263, 1167, 835, $685 \mathrm{~cm}^{-1}$; MS m/z: $398\left(\mathrm{M}^{+}, 0.14\right), 43$ (8.6), 77 (17), 92 (14), 107 (20), 135 (100), 191 (12). Anal. calcd for $\mathrm{C}_{23} \mathrm{H}_{26} \mathrm{O}_{6}$ : C 69.33, H 6.58; found C 69.27, H 6.61. 
4-(4'-三氟甲基)苯基-4-丁酮酸 [4-(4'-三氟甲基)苯甲 酰基]丁酯(4e): 白色晶体, 产率 73\%. m.p. $85 \sim 86{ }^{\circ} \mathrm{C} ;{ }^{1} \mathrm{H}$ NMR (400 MHz, $\left.\mathrm{CDCl}_{3}\right) \delta: 8.07$ (t, $\left.J=7.2 \mathrm{~Hz}, 4 \mathrm{H}\right), 7.73$ (d, $J=8.0 \mathrm{~Hz}, 4 \mathrm{H}), 4.17$ (t, $J=6.4 \mathrm{~Hz}, 2 \mathrm{H}), 3.33$ (t, $J=6.8$ $\mathrm{Hz}, 2 \mathrm{H}), 3.04$ (t, $J=6.8 \mathrm{~Hz}, 2 \mathrm{H}), 2.79$ (t, $J=6.4 \mathrm{~Hz}, 2 \mathrm{H})$, $1.84(\mathrm{~d}, J=7.6 \mathrm{~Hz}, 2 \mathrm{H}), 1.77(\mathrm{~d}, J=7.2 \mathrm{~Hz}, 2 \mathrm{H}) ;{ }^{13} \mathrm{C}$ NMR $\left(100 \mathrm{MHz}, \mathrm{CDCl}_{3}\right) \delta: 198.6,197.2,172.6,139.4$, 139.1, 128.3, 125.7, 64.3, 38.1, 33.6, 28.0, 20.3; IR (KBr) $v: 3055,1768,1646,1546,1484,1190,1068,768,688$, 646, $508 \mathrm{~cm}^{-1}$; MS m/z: 55 (4.3), 76 (1.2), 145 (36), 173 (100), 228 (11), 229 (1.2), 245 (0.76). Anal. calcd for $\mathrm{C}_{23} \mathrm{H}_{20} \mathrm{~F}_{6} \mathrm{O}_{4}: \mathrm{C} 58.23$, H 4.25; found C 58.30, H 4.29.

4-(4'-氯)苯基-4-丁酮酸 [4-(4'-氯)苯甲酰基]丁酯(4f): 白色晶体, 产率 64\%. m.p. 92 93 ${ }^{\circ} \mathrm{C}$; ${ }^{1} \mathrm{H}$ NMR (400 $\left.\mathrm{MHz}, \mathrm{CDCl}_{3}\right) \delta: 7.87(\mathrm{~d}, J=10.0 \mathrm{~Hz}, 4 \mathrm{H}), 7.38$ (d, $J=9.2$ $\mathrm{Hz}, 4 \mathrm{H}), 4.16$ (t, $J=6.4 \mathrm{~Hz}, 2 \mathrm{H}), 3.28$ (t, $J=6.4 \mathrm{~Hz}, 2 \mathrm{H})$, $2.98(\mathrm{t}, J=7.2 \mathrm{~Hz}, 2 \mathrm{H}), 2.75(\mathrm{t}, J=6.4 \mathrm{~Hz}, 2 \mathrm{H}), 1.82(\mathrm{t}$, $J=7.6 \mathrm{~Hz}, 2 \mathrm{H}), 1.74(\mathrm{t}, J=6.8 \mathrm{~Hz}, 2 \mathrm{H}) ;{ }^{13} \mathrm{C}$ NMR $(100$ $\left.\mathrm{MHz}, \mathrm{CDCl}_{3}\right) \delta: 198.4,196.8,172.8,139.6,139.4,131.8$, 129.4, 128.9, 64.3, 37.7, 33.2, 28.0, 20.4; IR (KBr) v: 3107, 3054, 2944, 1735, 1677, 1315, 1163, 978, 792, 524 $\mathrm{cm}^{-1}$; MS m/z: 50 (6.5), 75 (17), 111 (33), 139 (100), 194 (13), 195 (9.6), 211 (0.8). Anal. calcd for $\mathrm{C}_{21} \mathrm{H}_{20} \mathrm{Cl}_{2} \mathrm{O}_{4}$ : C 61.93, H 4.95; found C 61.96, H 4.96.

1,9-二苯基-1,5,9-壬三酮(7a): 白色晶体，产率 55\%. m.p. $67 \sim 68{ }^{\circ} \mathrm{C} ;{ }^{1} \mathrm{H}$ NMR $\left(400 \mathrm{MHz}, \mathrm{CDCl}_{3}\right) \delta$ : $7.97 \sim$ $7.92(\mathrm{~m}, 4 \mathrm{H}), 7.57 \sim 7.54(\mathrm{~m}, 2 \mathrm{H}), 7.47 \sim 7.26(\mathrm{~m}, 4 \mathrm{H})$, $3.04 \sim 2.97(\mathrm{~m}, 4 \mathrm{H}), 2.57 \sim 2.51(\mathrm{~m}, 4 \mathrm{H}), 2.06 \sim 1.99(\mathrm{~m}$, $4 \mathrm{H}) ;{ }^{13} \mathrm{C}$ NMR (100 MHz, $\left.\mathrm{CDCl}_{3}\right) \delta: 210.1,199.7,136.7$, 133.0, 128.6, 128.0, 41.6, 37.4, 18.2; IR (KBr) v: 3443, 3061, 2951, 2891, 1705, 1679, 1274, 1205, 1006, 734, 688 $\mathrm{cm}^{-1}$; MS m/z: $322\left(\mathrm{M}^{+}, 0.2\right), 217$ (3), 203 (9.1), 189 (4.2), 175 (26.9), 147 (43.3), 133 (10.8), 119 (22.2), 105 (100), 77 (37.5). Anal. calcd for $\mathrm{C}_{21} \mathrm{H}_{22} \mathrm{O}_{3}$ : C 78.23, $\mathrm{H}$ 6.88; found C 78.19, H 6.92 .

1,9-二(4'-甲基苯基)-1,5,9-壬三酮(7b): 白色晶体， 产率 53\%. m.p. 109 $110{ }^{\circ} \mathrm{C} ;{ }^{1} \mathrm{H}$ NMR (400 MHz, $\mathrm{CDCl}_{3}$ ) $\delta: 7.86 \sim 7.84(\mathrm{~m}, 4 \mathrm{H}), 7.26 \sim 7.24(\mathrm{~m}, 4 \mathrm{H}), 3.00 \sim 2.96$ (m, 4H), 2.56 2.52 (m, 4H), $2.41(\mathrm{~s}, 6 \mathrm{H}), 2.05 \sim 1.98(\mathrm{~m}$, $4 \mathrm{H}) ;{ }^{13} \mathrm{C}$ NMR $\left(100 \mathrm{MHz}, \mathrm{CDCl}_{3}\right) \delta: 210.2,199.4,143.8$, 134.3, 129.2, 128.1, 41.7, 37.3, 21.6, 18.3; IR (KBr) $v$ : 3443, 3056, 2936, 1670, 1674, 1605, 1409, 1181, 1001, $822,566,457 \mathrm{~cm}^{-1}$; MS m/z: $350\left(\mathrm{M}^{+}, 0.6\right), 231$ (1.1), 217 (9.1), 189 (19.1), 161 (28.5), 147 (9.7), 133 (2.1), 119 (100), 91 (36.1). Anal. calcd for $\mathrm{C}_{23} \mathrm{H}_{26} \mathrm{O}_{3}: \mathrm{C} 78.83, \mathrm{H}$
7.48; found C 78.78, H 7.51.

1,9-二(3'-甲基苯基)-1,5,9-壬三酮(7c)：白色晶体， 产率 54\%. m.p. $69 \sim 70{ }^{\circ} \mathrm{C} ;{ }^{1} \mathrm{H}$ NMR (400 MHz, $\left.\mathrm{CDCl}_{3}\right) \delta$ : $7.63(\mathrm{~d}, J=8 \mathrm{~Hz}, 2 \mathrm{H}), 7.38 \sim 7.34(\mathrm{~m}, 2 \mathrm{H}), 7.26 \sim 7.21(\mathrm{~m}$, $4 \mathrm{H}), 2.95 \sim 2.90(\mathrm{~m}, 4 \mathrm{H}), 2.55 \sim 2.49(\mathrm{~m}, 4 \mathrm{H}), 2.47(\mathrm{~s}$, $6 \mathrm{H}), 2.02 \sim 1.95(\mathrm{~m}, 4 \mathrm{H}) ;{ }^{13} \mathrm{C} \mathrm{NMR}\left(100 \mathrm{MHz}, \mathrm{CDCl}_{3}\right) \delta$ : 210.0, 203.7, 138.0, 137.7, 131.9, 131.2, 128.4, 125.7, 41.6, 40.3, 21.3, 18.3; IR (KBr) v: 3443, 2964, 2920, 2891, $1708,1676,1450,1417,1375,1266,1003,740 \mathrm{~cm}^{-1}$; MS $m / z: 350\left(\mathrm{M}^{+}, 0.2\right), 231(0.9), 217$ (3.5), 189 (5.6), 161 (12.7), 147 (6.2), 133 (1.0), 119 (100), 91 (30.6). Anal. calcd for $\mathrm{C}_{23} \mathrm{H}_{26} \mathrm{O}_{3}$ : C 78.83, H 7.48; found $\mathrm{C} 78.79, \mathrm{H}$ 7.42 .

1,9-二(2'-甲基苯基)-1,5,9-壬三酮(7d)：白色晶体， 产率 61\%; m.p. $52 \sim 53{ }^{\circ} \mathrm{C} .{ }^{1} \mathrm{H}$ NMR $\left(400 \mathrm{MHz}, \mathrm{CDCl}_{3}\right) \delta$ : $7.76 \sim 7.74(\mathrm{~m}, 4 \mathrm{H}), 7.36 \sim 7.27(\mathrm{~m}, 4 \mathrm{H}), 3.02 \sim 2.98(\mathrm{~m}$, $4 \mathrm{H}), 2.59 \sim 2.53(\mathrm{~m}, 4 \mathrm{H}), 2.39(\mathrm{~s}, 6 \mathrm{H}), 2.05 \sim 1.98(\mathrm{~m}$, $4 \mathrm{H}) ;{ }^{13} \mathrm{C}$ NMR $\left(100 \mathrm{MHz}, \mathrm{CDCl}_{3}\right) \delta: 210.2,199.9,138.3$, 136.8, 133.8, 128.5, 128.4, 125.2, 41.6, 37.5, 21.3, 18.2; IR (KBr) $v: 3445,2957,2929,2889,1697,1678,1582,1267$, 1181, 980, 793, $689 \mathrm{~cm}^{-1}$; MS m/z: $350\left(\mathrm{M}^{+}, 0.8\right), 231$ (0.9), 217 (7.4), 189 (17.1), 161 (28.8), 147 (9.3), 133 (0.9), 119 (100), 91 (37.9). Anal. calcd for $\mathrm{C}_{23} \mathrm{H}_{26} \mathrm{O}_{3}$ : C 78.83, H 7.48; found C 78.90, H 7.52.

1,9-二(4'-氯苯基)-1,5,9-壬三酮(7e): 白色晶体, 产 率 69\%. m.p. $116 \sim 117{ }^{\circ} \mathrm{C} ;{ }^{1} \mathrm{H}$ NMR (400 MHz, $\left.\mathrm{CDCl}_{3}\right) \delta$ : 7.89 (d, $J=8.8 \mathrm{~Hz}, 4 \mathrm{H}), 7.43$ (d, $J=8 \mathrm{~Hz}, 4 \mathrm{H}), 3.00 \sim 2.97$ (m, $4 \mathrm{H}), 2.57 \sim 2.53(\mathrm{~m}, 4 \mathrm{H}), 2.05 \sim 1.98(\mathrm{~m}, 4 \mathrm{H}) ;{ }^{13} \mathrm{C}$ NMR $\left(100 \mathrm{MHz}, \mathrm{CDCl}_{3}\right) \delta: 210.0,198.4,139.5,135.0$, 129.4, 128.9, 41.5, 37.4, 21.6, 18.1; IR (KBr) v: 3441, 2953, 2879, 1702, 1672, 1588, 1400, 1375, 1275, 1098, 1004, 822, 746, 571, $528 \mathrm{~cm}^{-1}$; MS m/z: $391\left(\mathrm{M}^{+}, 1.2\right)$, 251 (1.8), 237 (8.9), 209 (27.4), 181 (38.7), 167 (16.8), 153 (15.8), 139 (100), 111 (49.5). Anal. calcd for $\mathrm{C}_{21} \mathrm{H}_{20} \mathrm{Cl}_{2} \mathrm{O}_{3}$ : C 64.46, H 5.15; found C 64.51, H 5.19.

1,9-二(4'-三氟甲基苯基)-1,5,9-壬三酩(7f): 白色晶 体, 产率 75\%. m.p. 117 118 ${ }^{\circ} \mathrm{C} ;{ }^{1} \mathrm{H}$ NMR $(400 \mathrm{MHz}$, $\left.\mathrm{CDCl}_{3}\right) \delta: 8.06(\mathrm{~d}, J=8.4 \mathrm{~Hz}, 4 \mathrm{H}), 7.73(\mathrm{~d}, J=8 \mathrm{~Hz}, 4 \mathrm{H})$, $3.07 \sim 3.03(\mathrm{~m}, 4 \mathrm{H}),, 2.59 \sim 2.56(\mathrm{~m}, 4 \mathrm{H}), 2.07 \sim 2.00(\mathrm{~m}$, $4 \mathrm{H}) ;{ }^{13} \mathrm{C}$ NMR $\left(100 \mathrm{MHz}, \mathrm{CDCl}_{3}\right) \delta: 209.8,198.7,139.4$, 134.2, 128.4, 125.7, 125.6, 41.5, 37.7, 21.6, 18.0; IR (KBr) $v: 3442,2958,2887,1708,1687,1328,1273,1140,1065$, 1004, 827, $601 \mathrm{~cm}^{-1}$; MS m/z: $458\left(\mathrm{M}^{+}, 0.1\right), 285(1.0)$, 271 (4.7), 243 (29.1), 215 (62.5), 201 (9.8), 173 (100), 145 (46.1), 126 (3.6), 125 (4.1). Anal. calcd for $\mathrm{C}_{23} \mathrm{H}_{20} \mathrm{~F}_{6} \mathrm{O}_{3}$ : C 
60.26, H 4.40; found C 60.32, H 4.36.

1,9-二(2-呋喃基)-1,5,9-壬三酮(7g)：白色晶体，产 率 58\%; m.p. $77 \sim 78^{\circ} \mathrm{C}$; ${ }^{1} \mathrm{H} \mathrm{NMR}\left(400 \mathrm{MHz}, \mathrm{CDCl}_{3}\right) \delta$ : 7.55 (t, $J=0.8 \mathrm{~Hz}, 2 \mathrm{H}), 7.21(\mathrm{~d}, J=3.2 \mathrm{~Hz}, 2 \mathrm{H}), 6.54$ (q, $J=1.6 \mathrm{~Hz}, 2 \mathrm{H}), 2.88 \sim 2.84(\mathrm{~m}, 4 \mathrm{H}), 2.54 \sim 2.51(\mathrm{~m}, 4 \mathrm{H})$, $2.03 \sim 1.96(\mathrm{~m}, 4 \mathrm{H}) ;{ }^{13} \mathrm{C} \mathrm{NMR}\left(100 \mathrm{MHz}, \mathrm{CDCl}_{3}\right) \delta$ : 209.8, 188.9, 152.5, 146.3, 117.1, 112.2, 41.5, 37.2, 18.1; IR (KBr) v: 3445, 3117, 2953, 1701, 1666, 1467, 1419, 1398, 1280, 1036, 762, $594 \mathrm{~cm}^{-1}$; MS m/z: $302\left(\mathrm{M}^{+}, 0.4\right)$, 193 (11.6), 165 (51.1), 137 (82.6), 123 (13.8), 95 (100), 67(4.8). Anal. calcd for $\mathrm{C}_{17} \mathrm{H}_{18} \mathrm{O}_{5}$ : $\mathrm{C} 67.54, \mathrm{H} \mathrm{6.00}$; found C 67.49, H 5.96.

3,7,11-十三三酮(7h): 白色晶体，产率 27\%. m.p. $103 \sim 104{ }^{\circ} \mathrm{C} ;{ }^{1} \mathrm{H}$ NMR (400 MHz, $\left.\mathrm{CDCl}_{3}\right) \delta: 2.45 \sim 2.39$ $(\mathrm{m}, 12 \mathrm{H}), 1.87 \sim 1.80(\mathrm{~m}, 4 \mathrm{H}), 1.07 \sim 1.03(\mathrm{~m}, 6 \mathrm{H}) ;{ }^{13} \mathrm{C}$ NMR (100 MHz, $\left.\mathrm{CDCl}_{3}\right) \delta: 211.1,210.0,41.5,41.1,35.8$, 17.7, 7.7; IR (KBr) v: 3441, 2973, 1706, 1453, 1415, 1381, 1258, 1103, 1059, 1029, $771 \mathrm{~cm}^{-1}$; MS m/z: $226\left(\mathrm{M}^{+}, 0.1\right)$, 211 (0.2), 197 (7.5), 155 (10.0), 127 (47.1), 99 (100), 85 (31.6), 71 (9.5), 57(87.4). Anal. calcd for $\mathrm{C}_{13} \mathrm{H}_{22} \mathrm{O}_{3}$ : C 68.99, H 9.80; found C 69.03, H 9.85.

丙酸(4-氯)丁酯 $(8)$ : 无色油状液体, 产率 $68 \%$. ${ }^{1} \mathrm{H}$ NMR $\left(\mathrm{CDCl}_{3}, 400 \mathrm{MHz}\right) \delta: 4.09$ (t, $\left.J=12.4 \mathrm{~Hz}, 2 \mathrm{H}\right), 3.54$ $(\mathrm{t}, J=8.0 \mathrm{~Hz}, 2 \mathrm{H}), 2.34 \sim 2.30(\mathrm{~m}, 2 \mathrm{H}), 1.84 \sim 1.78(\mathrm{~m}$, $4 \mathrm{H}), 1.12(\mathrm{t}, J=8.4 \mathrm{~Hz}, 3 \mathrm{H}) ;{ }^{13} \mathrm{C} \mathrm{NMR}\left(\mathrm{CDCl}_{3}, 100 \mathrm{MHz}\right)$ $\delta: 174.2,63.1,44.1,28.8,27.1,25.7,8.7$.

\section{2 结果与讨论}

我们选择苯甲酰氯(12 mmol)和化合物 1 (6 mmol) 的双碘化锌试剂的反应为模板反应, 考察了 $\mathrm{CuCN}$ 和 $\mathrm{LiCl}$ 用量、反应温度对偶联反应的影响，结果见表 1 .

表 $1 \mathrm{CuCN}$ 和 $\mathrm{LiCl}$ 的用量及反应温度对偶联反应的影响 ${ }^{a}$ Table 1 Effect of amount of $\mathrm{CuCN}, \mathrm{LiCl}$ and reaction temperture on the coupling reaction

\begin{tabular}{ccccc}
\hline Entry & $\mathrm{CuCN} / \mathrm{mmol}$ & $\mathrm{LiCl} / \mathrm{mmol}$ & $\mathrm{Temp}^{\circ}{ }^{\circ} \mathrm{C}$ & Isolated yield $^{b} / \%$ \\
\hline 1 & 0 & 0 & -25 & 0 \\
2 & 0 & 24 & -25 & 0 \\
3 & 12 & 0 & -25 & 10 \\
4 & 12 & 24 & -25 & 54 \\
5 & 12 & 24 & 0 & 15 \\
6 & 12 & 24 & -15 & 45 \\
7 & 12 & 24 & -40 & 26 \\
\hline
\end{tabular}

${ }^{a}$ Reaction conditions: benzoyl chloride $(12 \mathrm{mmol}), 1(6 \mathrm{mmol})$ under nitrogen atmosphere; ${ }^{b}$ isolate yield.

实验结果表明, 当反应体系中没有 $\mathrm{CuCN}$ 和 $\mathrm{LiCl}$
存在或只有 $\mathrm{LiCl}$ 存在时，偶联反应不发生(表 1, Entries $1 \sim 2)$. 仅有 $\mathrm{CuCN}$ 存在时, 反应可以进行, 但产率只有 $10 \%$ (表 1, Entry 3). $\mathrm{CuCN}$ 和 $\mathrm{LiCl}$ 同时存在且物质的量 比为 $1: 2$ 时，偶联反应发生，且产物产率大大提高，达 到 54\%(表 1, Entry 4). 这说明双有机碘化锌活性较低, 不能直接与酰氯发生偶联反应. 只有在 $\mathrm{CuCN}$ 存在下, 它被转化为活性相对较高的铜锌试剂时才能与酰氯发 生反应，而 $\mathrm{LiCl}$ 则起到了活化酰氯的作用，有助于提高 产率 ${ }^{[10]}$.

另外, 温度对反应也有很大影响. 当温度高于 $0{ }^{\circ} \mathrm{C}$ 时，反应产物很复杂，无法分离得到目标产物；当温度 在 $0{ }^{\circ} \mathrm{C}$ 或 $-15{ }^{\circ} \mathrm{C}$ 时，产物产率为 $15 \%$ 和 $45 \%$ (表 1 , Entries $5 \sim 6$ ); 当温度降低至 $-25{ }^{\circ} \mathrm{C}$ 时，反应产率提高 到 $54 \%$ (表 1, Entry 4); 当温度再降低为 $-40{ }^{\circ} \mathrm{C}$ 时, 产率 只有 $26 \%$ (表 1, Entry 7). 这表明 $-25{ }^{\circ} \mathrm{C}$ 是偶联反应的 较佳温度.

反应溶剂对反应也有较大的影响. 我们选择了能溶 解 $\mathrm{CuCN}$ 和 $\mathrm{LiCl}$ 的溶剂 $\mathrm{DMF}$ 和 2-甲基四氢呋喃进行实 验，用 DMF 作溶剂时反应较复杂，未得到目标产物; 用 2-甲基四氢呋喃作溶剂时比 THF 更易开环，未得到目标 产物. 脂肪族酰氯易与 THF 反应开环成酯, 所以脂肪族 酰氯产率很低(以丙酰氯为例, 主要得到化合物 $\mathbf{8}$, Scheme 4). 当酰氯为芳香族酰氯时, THF 不易开环反应, 与双有机锌试剂反应均能给出较好结果.

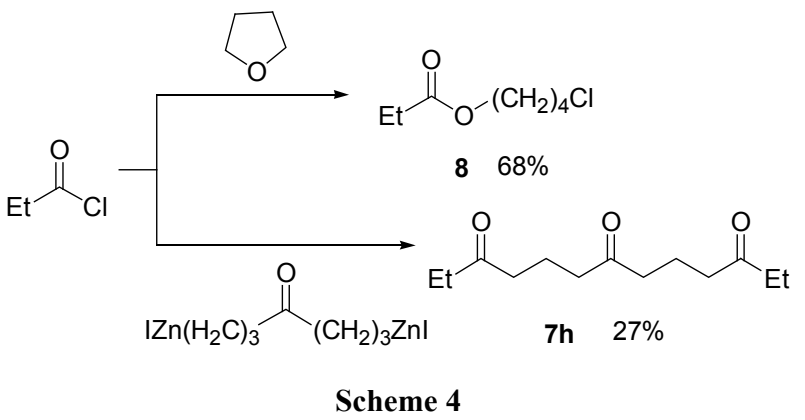

在最佳反应条件下, 我们对反应的底物范围作了考 察(表 2). 实验结果表明芳香族酰氯与双有机锌试剂反 应均能给出较好结果，尤其是芳环对位带有吸电子基的 芳香族酰氯产率较高.

综上所述，我们成功制备了带官能团的双有机碘化 锌试剂, 并研究了该试剂在 $\mathrm{CuCN} \cdot 2 \mathrm{LiCl}$ 存在下与芳基 酰氯的反应，得到含醚键、酯基以及羰基的二酮类化合 物。该方法条件温和，操作简便，产率中等，这为带官 能团的长链二酮类化合物的合成提供了一条简便方法, 拓展了有机卤化锌试剂在有机合成中的应用。 
表 2 带官能团的双有机碘化锌试剂与酰氯的偶联反应 ${ }^{a}$

Table 2 Coupling reaction of fuctionalized bisorganozinc iodides and acyl chlorides

\begin{tabular}{cclcc}
\hline Entry & Substrate & $\mathrm{R}$ & Product & Isolated yield ${ }^{b} \%$ \\
\hline 1 & $\mathbf{1}$ & $\mathrm{H}$ & $\mathbf{2 a}$ & 54 \\
2 & $\mathbf{1}$ & $3-\mathrm{Me}$ & $\mathbf{2 b}$ & 55 \\
3 & $\mathbf{1}$ & $2-\mathrm{Me}$ & $\mathbf{2 c}$ & 52 \\
4 & $\mathbf{1}$ & $4-\mathrm{Me}$ & $\mathbf{2 d}$ & 53 \\
5 & $\mathbf{1}$ & $3-\mathrm{OMe}$ & $\mathbf{2 e}$ & 52 \\
6 & $\mathbf{1}$ & $4-\mathrm{CF}_{3}$ & $\mathbf{2 f}$ & 66 \\
7 & $\mathbf{3}$ & $\mathrm{H}$ & $\mathbf{4 a}$ & 52 \\
8 & $\mathbf{3}$ & $3-\mathrm{Me}$ & $\mathbf{4 b}$ & 58 \\
9 & $\mathbf{3}$ & $4-\mathrm{Me}$ & $\mathbf{4 c}$ & 56 \\
10 & $\mathbf{3}$ & $3-\mathrm{OMe}$ & $\mathbf{4 d}$ & 52 \\
11 & $\mathbf{3}$ & $4-\mathrm{CF}_{3}$ & $\mathbf{4 e}$ & 73 \\
12 & $\mathbf{3}$ & $4-\mathrm{Cl}$ & $\mathbf{4 f}$ & 64 \\
13 & $\mathbf{6}$ & $\mathrm{Ph}_{1}$ & $\mathbf{7 a}$ & 55 \\
14 & $\mathbf{6}$ & $4-\mathrm{MeC}_{6} \mathrm{H}_{4}$ & $\mathbf{7 b}$ & 53 \\
15 & $\mathbf{6}$ & $3-\mathrm{MeC}_{6} \mathrm{H}_{4}$ & $\mathbf{7 c}$ & 54 \\
16 & $\mathbf{6}$ & $2-\mathrm{MeC}_{6} \mathrm{H}_{4}$ & $\mathbf{7 d}$ & 61 \\
17 & $\mathbf{6}$ & $4-\mathrm{ClC}_{6} \mathrm{H}_{4}$ & $\mathbf{7 e}$ & 69 \\
18 & $\mathbf{6}$ & $4-\mathrm{CF}_{3} \mathrm{C}_{6} \mathrm{H}_{4}$ & $\mathbf{7 f}$ & 75 \\
19 & $\mathbf{6}$ & $2-\mathrm{Furyl}$ & $\mathbf{7 g}$ & 58 \\
20 & $\mathbf{6}$ & $\mathrm{Et}^{2}$ & $\mathbf{7 h}$ & 27 \\
\hline
\end{tabular}

${ }^{a}$ Reaction conditions: benzoyl chloride $(12 \mathrm{mmol}), \mathbf{1}$ or 3 or $\mathbf{6}(6 \mathrm{mmol})$ under nitrogen atmosphere at $-25{ }^{\circ} \mathrm{C} .{ }^{b}$ isolate yield.

\section{References}

[1] Frankland, E. Liebigs Ann. Chem. 1849, 71, 171.

[2] Negishi, E. Acc. Chem. Res. 1982, 15, 340.

[3] (a) Furstner, A. Synthesis 1989, 571.

(b) Knochel, P.; Almeana Perea, J. J.; Jones, P. Tetrahedron 1998, 54,8275 .

[4] Knochel, P.; Yeh, M. C. P.; Berk, S. C.; Talbert, J. J. Org. Chem. 1988, 53, 2390.

[5] Hunsdiecker, H.; Erlbach, H.; Vogt, E. DE 722467, 1942 [Chem. Abstr. 1943, 37, P 5080].

[6] Wittig, G.; Jautelat, M. Liebigs. Ann. Chem. 1967, 702, 24.

[7] (a) Sato, T.; Soai, K.; Suzuki, K.; Mukaiyama, T. Chem. Lett. 1978, 601.

(b) Mukaiyama, T.; Soai, K.; Sato, T.; Shimizu, H.; Suzuki, K. J. Am. Chem. Soc. 1979, 101, 1455.
[8] (a) Rossiter, B. E.; Swingle, N. M. Chem. Rev. 1992, 92, 771.

(b) Yamaguchi, M.; Shiraishi, T.; Hirama, M. Angew. Chem., Int. Ed. Engl. 1993, 32, 1176.

(c) Sasai, H.; Arai, T.; Shibasaki, M. J. Am. Chem. Soc. 1994, 116, 1571.

(d) Wang, Y.; Gladysz, J. A. J. Org. Chem. 1995, 60, 903.

(e) Yamaguchi, M.; Igarashi, Y.; Reddy, R. S.; Shiraishi, T.; Hirama, M. Tetrahedron 1997, 53, 11223.

(f) Soai, K.; Niwa, S. Chem. Rev. 1992, 92, 833.

(g) Knochel, P.; Vettel, S.; Eisenberg, C. Appl. Organomet. Chem. 1995, 9, 175 .

[9] (a) Negishi, E.; Valente, L. F.; Kobayashi, M. J. Am. Chem. Soc. 1980, 102, 3298.

(b) Kobayashi, M.; Negishi, E. J. Org. Chem. 1980, 45, 5223.

(c) Negishi, E. Acc. Chem. Res. 1982, 15, 340.

(d) Negishi, E.; Bagheri, V.; Chatterjee, S.; Luo, F.-T.; Miller, J. A.; Stoll, A. T. Tetrahedron Lett. 1983, 24, 5181.

[10] AchyuthaRao, S.; Knochel, P. J. Org. Chem. 1991, 56, 4591.

[11] (a) Wang, J.-X.; Fu, Y.; Hu, Y. Angew Chem., Int. Ed. 2002, 41, 2757.

(b) Hu, Y.; Yu, J.; Yang, S.; Wang, J.-X.; Yin, Y. Synlett 1998, 1213.

(c) Hu, Y.; Wang, J.-X.; Li, W. Chem. Lett. 2001, 174.

(d) Wang, J.-X.; Fu, Y.; Hu, Y.; Wang, K. Synthesis 2003, 1506.

(e) Wang, J.-X.; Wang, K.; Zhao, L.; Li, H.; Fu, Y.; Hu, Y. Adv. Synth. Catal. 2006, 348, 1262;

(f) Huang, D.; Wang, J.-X. Synlett 2007, 2272.

(h) Fu, Y.; Wang, J.-X.; Wang, K.; Hu, Y. Tetrahedron 2008, 64, 11124.

(g) Wei, Y.-J.; Ren, H.; Wang, J.-X. Tetrahedron Lett. 2008, 49, 5697.

[12] Xu, C. M.; Yang, L.; Huang, D. F.; Niu, T.; Hu, Y. L. Chin. J. Org. Chem. 2010, 30, 1240 (in Chinese)

(徐长明, 杨否, 黄丹凤, 牛腾, 胡雨来, 有机化学, 2010, 30, 1240.)

[13] (a) Hu, Y. L. Ph.D. Dissertation, Lanzhou Institute of Chemical Physics, Chinese Academy of Sciences, Lanzhou, 1998 (in Chinese).

(胡雨来, 博士论文, 中国科学院兰州化学物理研究所, 兰州, 1998.)

(b) Hart, H.; Curtis, O. E. J. Am. Chem. Soc. 1956, 78, 112.

[14] (a) Knochel, P.; Yeh, M. C. P.; Berk, S. C.; Talbert, J. J. Org. Chem. 1988, 53, 2390.

(b) Picontin, G.; Miginiac, P. J. Org. Chem. 1987, 52, 4796. 